\title{
LA CLASSE MEDIA BRASILIANA E L'ARCHETIPO DEL PREGIUDIZIO DI CLASSE
}

\section{ARTICOLO ORIGINALE}

GIESBRECHT, Daniel Florence ${ }^{1}$

GIESBRECHT, Daniel Florence. La classe media brasiliana e l'archetipo del pregiudizio di classe. Revista Científica Multidisciplinar Núcleo do Conhecimento. Anno 05, Ed. 02, Vol. 04, pp. 104-124. febbraio 2020. ISSN: 2448-0959, Collegamento di accesso: https://www.nucleodoconhecimento.com.br/storia/archetipo-di-pregiudizio

\section{RIEPILOGO}

Questo articolo ha lo scopo di elaborare una ricostituzione storica, dal seriale a lungo termine, la formazione della classe media brasiliana e anche il suo archetipo di pregiudizio di classe. Abbiamo usato come punto di partenza per la nostra riflessione il fatto che il Brasile ha vissuto più di trecento anni di schiavitù, che ha fatto la profusione di un immaginario razzista, con conseguente pregiudizio e naturalizzato alle popolazioni afrodiscendenti, oltre ad essersi esteso al povero cittadino, in generale. Cerchiamo di mettere in relazione in modo storicista gli oggetti studiati ai concetti sociologici della socializzazione, oltre a caratterizzare le idee borghesi della classe media e delle élite brasiliane dalla storia delle mentalità. Intendiamo contribuire a una migliore comprensione degli ostacoli creati dalla mancanza di pratiche di alterità nelle relazioni sociali quotidiane.

Parole chiave: Classe sociale, pregiudizio, schiavitù, esclusione sociale, alterità.

\footnotetext{
${ }^{1}$ Dottorato di ricerca in Storia Contemporanea; Master in Educazione; Laurea in Scienze Sociali, Laurea in Pedagogia, Laurea in Storia.
} 


\section{INTRODUZIONE}

Quando abbiamo studiato gli indicatori economici sul potenziale brasiliano, abbiamo notato numeri costanti e alti, molto competitivi rispetto ad altri paesi, tanto che il Brasile, dagli anni ' 60 ad oggi, è sempre tra i quindici paesi con il prodotto interno lordo più alto del mondo.

I numeri non si ripetono se cerchiamo dati di riferimento sui tassi di povertà e disuguaglianza. Realtà allarmanti della distribuzione del reddito, degli alti tassi di violenza, della mortalità infantile, tra gli altri, sono facilmente percepibili agli occhi di chi viaggia attraverso il vasto territorio brasiliano, dando l'impressione di passeggiare attraverso due mondi completamente diversi: quello dell'opulenza e quello della miseria, un'illusione di ottica di un'economia inserita con successo nello scenario mondiale, ma che maschera una complessa realtà sociale: quella dell'opulenza e quella della miseria, un'illusione di ottica di un'economia inserita con successo nello scenario mondiale, ma che maschera una complessa realtà sociale, che presenta, nella sua struttura, segni di profonda disuguaglianza.

Useremo la parola "povertà" per riferirci alla situazione di milioni di persone che vivono in Brasile. Ma il concetto di povertà, a seconda del contesto utilizzato e del suo factoring semantico, diventa complesso e polisemico. È da questa complessità semantica che proponiamo di dedurre la nostra riflessione attraverso questo articolo, mettendo in relazione la povertà con l'archetipo della formazione della classe media brasiliana e delle sue interrelazioni di classe.

Nell'immaginario intrinseco della classe media brasiliana nei suoi circoli di socializzazione, l'immaginario della povertà è trasfigurato in una percezione così semplificata della realtà che li circonda, al punto che l'individuo della classe subalterna, spesso in uno stato di insicurezza, acquisisce inconsciamente il discorso della classe a cui non appartiene e a cui viene sfruttata. In questa situazione, non è difficile imbattersi in frasi quotidiane del più puro buon senso che cercano, in ogni momento, una spiegazione per i mali delle disuguaglianze del paese: "Egli è povero perché è un 
vagabondo", "Povero non ama lavorare" o, ancora, "Poveri non sanno fare nulla e non possono imparare nulla".

Frasi come queste finiscono per produrre una naturalizzazione del sociale, cioè si aggrappa al carattere storico e sociale di un fatto riducendolo ad un evento naturale. Così, qualcosa di simile la mancanza cessa di essere visto come risultato di una struttura assemblata a priori, che finisce per legittimare le disuguaglianze nella sua prassi, per essere considerata un attributo personale di un individuo.

Prendendo in considerazione l'idea della naturalizzazione delle disuguaglianze e dell'individualizzazione delle aspettative e dei fallimenti ad hoc, è necessaria una riflessione accurata dei fattori di classe che contribuiscono alla naturalizzazione dell'archetipo di inferiorizzazione dei gruppi sociali in svantaggio e che rompa i modelli preconcetti dall'avvento del positivismo storico dell'Ottocento che, trasformando le scienze sociali in natura governate da leggi immutabili che sfuggono al controllo umano che sfuggono al controllo umano che sfuggono al controllo umano che sfuggono al controllo umano che sfuggono al controllo umano che sfuggono al controllo umano che sfuggono al controllo umano che sfuggono al controllo umano che sfuggono al controllo umano che sfuggono al controllo umano che sfuggono al controllo umano che sfuggono al controllo umano che sfuggono al controllo umano che sfuggono al controllo umano che sfuggono al controllo umano che sfuggono al controllo umano che sfuggono al controllo umano che sfuggono al controllo umano che sfuggono al controllo umano che sfuggono al controllo umano che sfuggono al controllo umano che sfugge al controllo umano che sfugge al controllo umano che sfugge al controllo umano che sfugge al controllo umano che sfugge al controllo umano che sfuggono al controllo umano che sfuggono al controllo umano che sfuggono al controllo umano che , indurre il conformismo, l'accettazione passiva e rassegnata di ciò che è storicamente e socialmente prodotto (Sucupira, 1984). L'archetipo dei poveri che falliscono quotidianamente, così radicato nell'immaginario collettivo brasiliano dalla colonia alla contemporaneità, è in questo senso un'operazione ideologica, che serve a mantenere un certo ordine sociale, cioè una configurazione della vita economica che va a vantaggio delle classi sociali che producono questa ideologia. 
Utilizzeremo prima il quadro teorico della Souza (2019), che sembrava più pertinente alla critica dei paradigmi che guidano il pensiero sociale brasiliano, in particolare quello del "culturalismo", nei suoi tentativi di spiegare le origini delle disuguaglianze nel paese. È nostra intenzione anche introdurre al lettore le idee che difenderemo a posteriori, in particolare che la strutturazione dell'archetipo mentale della classe media brasiliana è uno dei principali ostacoli alla costruzione di un paese che consolida $i$ valori repubblicani e che crea le condizioni per l'espansione dei diritti di cittadinanza per milioni di suoi abitanti.

\section{POLISSEMIA DI POVERTY}

La povertà è un fenomeno storico e sociale. Ciò significa che è strettamente legato alla struttura della società e al modo in cui ogni classe sociale si appropria dei beni prodotti o della ricchezza generata. Non è un concetto facile, ma possiamo usare criteri diversi per cercare di definirlo.

Il criterio del reddito personale o familiare è stato il più utilizzato in Brasile, ma riteniamo che sia impreciso, a causa di molti fattori che sono persino peggiorati negli ultimi anni, come la crescita di attività informali, che impedisce un controllo efficace del reddito[2].

Oltre al reddito, un altro criterio utilizzato per configurare la povertà è quello di verificare ciò che è considerato essenziale per la riproduzione della vita. Questo criterio, quindi, a nostro avviso, è anche riduzionista perché, a causa delle convenzioni internazionali, riguarda la povertà solo al consumo alimentare.

Sembra più pertinente esplodare il concetto ai "bisogni di base", che non sarebbero limitati solo al cibo, ma includerebbero altri aspetti considerati indispensabili per la riproduzione materiale dell'esistenza, ampliando l'idea di povertà per l'incapacità di qualcuno o di una famiglia di avere mezzi che consentano l'accesso a una serie di oggetti considerati essenziali per la sopravvivenza. Questo sarebbe configurato anche se la persona o la famiglia è esclusa dal sistema educativo, dal momento che la scolarizzazione oggi è essenziale per entrare nel mercato del lavoro. Secondo gli studi di Rocha (2006), è stato solo con l'espansione di questi concetti che abbiamo potuto 
comprendere un paese di dimensioni continentali come il Brasile, immerso in enormi disparità regionali.

La nostra idea argomentativa è quella di superare esattamente la visione materiale della costituzione del concetto di povertà per una valorizzazione psicologica e strutturale astratta e inconscia che, nella costruzione storica brasiliana, legittima questo concetto ancorato in un quadro metodologico sostenuto dall'archetipo del pregiudizio, che inferiorizza i gruppi sociali costituiti in un paese che aveva, per più di trecento anni, i segni quotidiani di un'economia e di una società orientate a schiavi.

II pregiudizio può servire interessi materiali diversi, che possono essere: classe, genere, politico, razziale o etnico e religioso. Ma per dimostrare la costituzione del pregiudizio naturalizzato dalla classe media brasiliana, abbiamo indubbiamente bisogno di conoscere le strutture storiche che sintetizzato, attraverso il passato precapitalista, le distanze tra i gruppi sociali di una società che, dal bipolarismo tra voi e lo schiavo, si muoveva verso un'organizzazione più complessa con l'avvento del capitalismo commerciale e industriale, ma che ha sempre mantenuto l'archetipo del passato schiavo.

La discriminazione e il pregiudizio possono anche produrre sentimenti di rassegnazione e conformità nelle vittime, che accettano di essere oppresse e sfruttate, come Sartre (1905-1980) ha avvertito in una commedia intitolata The Respectful Prostitut[3]e (1946). Ci sembra il caso brasiliano, con atteggiamenti quotidiani della sua classe media di inferiorizzazione dei meno favoriti, facendoli intromettere il proprio pregiudizio e l'idea di fallimento, credendo di meritare tali discriminazioni.

\section{ORIGINS HISTORICAL Di PREJUDICEAMENTI DELLA CLASSE MEDIA BRASILIANA}

Tra il XVI e il XIX secolo, il commercio di schiavi africani verso l'America collegava i due continenti a quello che sarebbe diventato il commercio più redditizio della storia brasiliana. Gli schiavi africani erano presenti nelle più diverse attività quotidiane. La naturalizzazione della schiavitù è diventata qualcosa di così radicato nelle strutture 
della società che è molto improbabile che sarebbe immaginato senza le tradizionali divisioni di coloro che hanno comandato e obbedito. Questo modello autoritario delle relazioni sociali consente di comprendere la permanenza simbolica praticata contro le popolazioni più povere, anche dopo l'abolizione della schiavitù alla fine del XIX secolo.

Con lo sviluppo del pensiero sociale brasiliano, soprattutto dall'inizio del XX secolo, abbiamo cercato di spiegare il quadro dicotomo che sperimenta dalla società nativa nel corso della sua storia. Crediamo che, per questo compito, la teoria più appropriata, promuovendo una migliore comprensione della costruzione di questa struttura sociale, sia quella della storia seriale, presente nelle metodologie di fernand Braude[4]l. Contemplare un'analisi semiotica della formazione delle classi sociali brasiliane è possibile solo quando si percepisce il seriale della lunga durata della schiavitù, che ha convalidato nella mentalità quotidiana del paese l'archetipo di pregiudizio, distacco e segregazione, da casa-grande a senza la, che, con la transizione alla modernità della fase mercantile e industriale del capitalismo, ha corroborato una nuova forma di sfruttamento, ma con l'abbigliamento strutturale del vecchio, segregando ricchi e poveri all'interno delle grandi città, così come nei circoli sociali.

Vale anche la pena ricordare che lo sviluppo di classi in Brasile, secondo Fausto (1997), è legato al consolidamento di una classe proprietaria, che possiede la proprietà fondiaria e altri mezzi di produzione. In un paese che un tempo era fondamentalmente agricolo, questa classe si appropriava facilmente la maggior parte della ricchezza prodotta, poiché sfruttava il lavoro schiavizzato.

Sotto l'egida della società coloniale, si è sviluppata in modo molto particolare nelle regioni in cui il sistema delle piantagioni è stato consolidato, relazioni sociali al di fuori della schiavitù tra liberati e proprietari. Con l'assenza di un sistema coloniale diretto, oltre ad un'immensità territoriale, che ostacolava l'attuazione di forme ottimizzate di controllo sotto la tutela della corona portoghese, e la creazione del sistema di distribuzione delle capitanerie ereditarie - si è sviluppato un tentativo di conciliazione pubblico-privato nel processo di colonizzazione - sono state sviluppate forme ambigue, contraddittorie e deregolamentate di relazioni tra individui di diverse classi. $\grave{E}$ in questo scenario che troviamo la cifra dell'aggregato. 
Questo strato intermedio di persone formalmente libere, tra padroni e schiavi, è formato da uomini e donne dipendenti, sia materialmente che simbolicamente di proprietari terrieri e persone. Nel senso più economico e materiale, la dipendenza di questo estratto sociale è inesorabile. (...) Questo costituisce tra noi - come forma economica, politica e sociale la figura dell'"aggregato". Figura fondamentale nella letteratura brasiliana e nella sociologia storica, l'aggregato formerà la prima classe intermedia tra proprietari e espropriati. (Sousa, 2018: 65-66).

Anche se ancora lontano dall'emergere della moderna classe media brasiliana, il gruppo di famiglie, anche dipendenti e spesso ispessimento le statistiche della popolazione espropriata - la perdita della protezione del loro "padrino" era sufficiente - è di fondamentale importanza comprendere l'introiezione mentale del pregiudizio tra le future classi sociali del Brasile moderno, dato che queste, allinterno di un sistema di dominazione autoritaria del potere privato sul pubblico, fu proprio la casta a naturalizzare i principi di controllo e violenza praticati in un certo senso, in una certa misura, sadico dalle élite ruraliste che lavoravano come schiavi, a delegare anche agli aggregati i diritti di opprimere quelli visti come "strumenti che parlavano", bestializzati e disumanizzati.

II fatto che ci interessi da qui è renderci conto che è stato attraverso questo rapporto di interdipendenza tra signori e aggregati, oltre al monopolio della forza stabilita sotto lo Stato come organizzatore della società, che il sentimento di disprezzo per l'altro era quello di portare alla costituzione di un comportamento sadico con gli schiavi e i più umili. II piacere della violenza è comunemente verificato nella storia di queste classi intermedie nella società brasiliana, al punto di essere più intensi - noi percepiamo il sadis[5]mo come una forma di riconoscimento di gruppo, o superiorità sull'altro - come una pratica quotidiana di coloro che chiamiamo uomini liberi. Scene di umiliazione, scherzi, satire e comportamenti ottusi faranno parte della portata del trattamento perpetrato sugli emarginati, che chiameremo "scum" sociale.

Dall'inizio dell'attività mineraria nel crepuscolo del XVII secolo e per tutto il XVIII secolo, il Brasile ha sperimentato il fenomeno dell'urbanizzazione, concentrato principalmente 
nella fascia di capitano di Minas Gerais. La società stratificata dello zucchero nordorientale era quella di un carattere più dinamico e poliedrico a Vila Rica. La schiavitù e la libertà andarono di pari passo in un'economia interdipendente, ma fornivano nuove forme di ascensione sociale, comprese le fodere nere ${ }^{[6]}$. L'esperienza mineraria è stata particolare, in cui metizo, neri e indiani hanno avuto un momento unico in cui potevano mettere in pratica per la prima volta il loro talento, da qui il gran numero di artisti, artigiani, commercialisti e l'apparizione di diverse altre professioni nella regione. Per la prima volta nella storia del Brasile, una conoscenza tecnica più espressiva sembrava differenziarsi da una società costruita da lavoro "grezzo" e manuale, spianando la strada all'emergere della classe media moderna.

All'inizio del XIX secolo fu il presagio di nuove arie che avrebbero scosso il continente europeo - e la sua più grande tempesta fu Napoleone Bonaparte. Le guerre iniziate dall'imperatore francese furono associate, oltre alle sue ambizioni megalomaniche, con la nuova forma assunta dal sistema capitalista nel suo stadio industriale. La Francia, il nemico storico della Gran Bretagna, stava iniziando un conflitto di proporzioni continentali per conquistare la sua giusta quota, in un presagio di quello che sarebbe stato il fenomeno imperialista ancora da innescare a metà del secolo. Questo fatto avrebbe un impatto diretto e indiretto sul futuro del Portogallo e di conseguenza brasile, perché la struttura amministrativa metropolitana avrebbe attraversato i mari per stabilirsi nella colonia.

Questo processo, che la famiglia reale portoghese si è consolidata, era già previsto alla scoperta delle miniere, alla presenza di alcune città coloniali di espressione, nella necessità di una maggiore vigilanza sulla ricchezza appena scoperta e di un maggiore controllo, da allora in poi, sul familismo e sul mandonoismo privato. Un esempio sintomatico del passaggio del potere dalla campagna alle città è il caso dei debiti dei patriarchi rurali precedentemente incolmabili, da allora in poi pagati sotto le forze di polizia. Altrettanto importante quanto il passaggio dal centro economicamente dinamico del Nordest al Sud-Est è stata la trasformazione sociale di grandi proporzioni, implicando nuove abitudini, 
nuovi ruoli sociali, nuove professioni e, alla fine, la costruzione di una nuova gerarchia sociale. (Souza, 2019: 61).

L'urbanizzazione alla fine ha rappresentato un ridimensionamento delle strutture di potere, facendola passare dal patriarcalismo individuale nelle mani dello stato che è stato organizzato. Da un punto di vista weberiana, il legittimo monopolio della violenza ha cominciato a concentrarsi sulle mani dell'apparato statale - come la polizia, per esempio - fondendosi con le prerogative delle antiche forme di autoritarismo di classe, ma mantenendo la pratica del sadismo, precedentemente privatamente, ora perpetrato dalle forze pubbliche.

Con l'apertura dei porti alle nazioni amiche nel 1808, decretata dall'allora principe reggente $\mathrm{D}$. Joo $\mathrm{VI}$, e con la firma dei trattati commerciali e di navigazione del 1810, il Brasile avrebbe sperimentato trasformazioni che avrebbero immediatamente avuto un impatto sulla sua struttura coloniale. L'ambiente delle città portuali, principalmente Rio de Janeiro, Salvador e Recife divennero, durante la notte, centri commerciali affollati, con l'ingresso di prodotti sconosciuti fino ad allora, che ha incoraggiato la formazione di una struttura monetaria. Gli uffici di scambio ed esportazione, le agenzie di importazione ed esportazione, le banche, insomma, una moltitudine di attività finora inutili nel mondo del patriarcato rurale, vissute solo nelle loro giuste proporzioni nelle regioni minerarie[7].

Ma la grande trasformazione sociale provocata da questo nuovo scenario europeo di relazioni economiche sarebbe l'ingresso di migliaia di immigrati. Oltre ai circa 15.000 portoghesi che arrivarono insieme la famiglia reale, anche l'inglese, il tedesco e il francese arrivarono in Brasile, per ragioni che vanno dall'economia all'arte. II futuro paese che sarebbe nato nel 1822 già segnato le caratteristiche di uno stato indipendente.

Gli immigrati erano manodopera libera che, si aggiungeva ai lavoratori liberi nati in Brasile, avrebbe costituito la classe media moderna di cui abbiamo parlato prima. Una classe diversa dalla vecchia famiglia rurale, che occupa la parte intermedia della gerarchia sociale, ma senza la necessità della protezione del suo compagno. I principi 
liberali e l'individualismo, che soffiavano fin dal XVIII secolo con la filosofia illuminista, entrarono nell'inconscio dell'abitante urbano. Una sorta di uomo self-made dei tropici avrebbe popolato l'immaginario della città.

Con il capitalismo mercantile apre un vuoto fondamentale per il suo mantenimento e la sua riproduzione: la necessità di capitale culturale per gestirlo. Le attività commerciali, manifatturiere, burocratiche e didattiche - per essere in alcuni esempi richiedono una forma di conoscenza tecnica e utilitaristica per il loro funzionamento, un fatto che contribuirebbe all'opposizione del lavoro manuale, finora comunemente svolto dallo schiavo o dai più poveri. La classe media sarà il gruppo che si appropria di questa conoscenza e, attraverso di essa, prenderà le distanze dai più umili, quelli del lavoro pesante, dal livello delle bestie.

Agli abbandonati e dimenticati, i compiti squalificati, tipici della plebaglia, rimangono. Questi, in larga misura, come nel caso dei lavori domestici, saranno una continuazione della schiavitù, ora sotto nuove maschere. Lo sfruttamento diretto dell'energia muscolare - che ci distingue a malapena da un cavallo o da un mulo - con poca conoscenza incorporata, è il caratteristico, se non l'unico, caratteristico in relazione ai lavoratori con un più alto grado di qualificazione tecnica. (Souza, 2018: 74).

Sia questa nuova classe media, sia la futura classe operaia minimamente specializzata, che chiameremo "bassa classe media", o "proletaria", cercherà a tutti i costi di differenziarsi dalla popolazione vista come "scum", formata principalmente da neri e meticci. Poiché non hanno i mezzi di produzione, cercheranno almeno di imitare i valori simbolici delle élite, riflettendo sull'ossessione per l'europeizzazione dei costumi e, attraverso l'educazione formale e tecnica, nel distacco dal lavoro manuale, così degradato dai valori occidentali. Qui abbiamo la nostra prima manifestazione concreta di pregiudizio incastonata dalla moderna classe media del XIX secolo.

Le questioni endogene ed esogene in relazione alla questione degli schiavi brasiliani furono determinanti per la maturazione iniziale del processo di modernizzazione dei rapporti di lavoro e della maturazione del capitalismo industriale nel XIX secolo. 
Dall'indipendenza brasiliana, I'Inghilterra ha esercitato pressioni sul neonato stato nazionale per dimostrare gli impegni per la graduale estinzione del commercio degli schiavi praticato dalla metà del XVI secolo. I movimenti interni guidati da intellettuali liberali, come l'élite del caffè stessa, discutevano apertamente l'abolizione.

Tali fatti, in un primo momento, potrebbero sottolineare in modo sui generis che il paese, al suo ritmo, potrebbe consolidare la sua transizione dal capitalismo commerciale al capitalismo industriale, perché lo scenario che si apriva con l'abolizione della schiavitù sposterebbe non solo un'enormità di capitale nei settori produttivi, ma anche l'ammodernamento delle relazioni di classe, superando gli amalgami del sistema schiavo obsoleto. Ma non è quello che è successo. L'industrializzazione si sarebbe consolidata solo nel secolo successivo, sotto la direzione dello stato, nei governi Getilo Vargas e Juscelino Kubitschek, così come nel periodo dittatoriale. Superare la schiavitù, nel suo campo simbolico, non sarebbe mai stato superato.

Anche con l'abolizione consumata nel 1888, un fatto che ha reso il Brasile l'ultimo paese al mondo a porre fine alla schiavitù formale, lo scenario perverso della violenza fisica e simbolica è continuato. Inoltre, i neri liberati incontrarono enormi difficoltà nel loro inserimento nel mercato del lavoro, poiché dovevano ancora competere con la forza lavoro immigrata, ora deliberatamente stimolata come pratica statale. Fu all'interno di questo rapporto perverso di permanenza che, nel corso del tempo, si formò il Brasile, in concomitanza con il processo di urbanizzazione, una classe della popolazione che era completamente esclusa dai principi fondamentali della cittadinanza, [8]e questo avrebbe colpito anche le sue prossime generazioni, un effetto che chiameremo la "palla di neve nuvolos[9]a".

\section{ESCLUSI L'INDUSTRIALIZZAZIONE E IL BOURGEOIS IMAGINARY DELLA CLASSE DI ME}

Il vecchio ordine razziale non scomparve con la fine del lavoro degli schiavi. Invece, è stato assimilato e integrato nelle nuove strutture sociali derivanti dallo sviluppo del libero lavoro. Secondo Fernandes (1974), la rivoluzione borghese innescata con la fine della schiavitù avvantaggiò solo alcuni segmenti della società brasiliana. 
Tra i più favoriti c'era la parte "bianca" della popolazione di origine europea, corroborando le teorie dello sbiancamento in voga nel paese. Questo gruppo ha beneficiato di tutte le trasformazioni derivanti dalla crescita economica, dal scatto industrializzato, dal processo di urbanizzazione e dallo sviluppo di una cultura urbanistica. La classe emarginata, composta da afrodiscendenti, rimase ai margini della società nazionale, esclusa in gran parte da tutti i miglioramenti che queste trasformazioni producevano. Questi fatti hanno sottolineato che la disarticazione del lavoro schiavo negli ultimi decenni del XIX secolo non è stata accompagnata da cambiamenti sostanziali nel vecchio ordine razziale, caratterizzato da pregiudizi e discriminazioni. L'emarginazione sociale di circa il 50\% dei brasiliani ha chiaramente evidenziato i limiti della modernizzazione economica del paese.

Con il mantenimento dei mezzi di produzione nelle mani dell'agro-exportelite e della classe industriale incipiente, fu nella città e nel suo ambiente cosmopolita che si consolidò lo stile di vita borghese e, insieme ad esso, tutto il suo simbolismo. Fu con l'emergere della classe media urbana, in particolare quella incentrata sulle attività intellettuali e finanziarie, che si diffuse il senso di europeizzazione e le dogane. L'incorporazione dei valori aristocratici europei può essere percepita nell'adozione di un modello di gusto e di lusso che cercasse di eguagliare quello degli strati aristocratici. In una sorta di "cannibalismo simbolico", la classe media più ricca introionò pregiudizi e patriarcati, ma con un abbigliamento che si diceva fosse moderno.

In una dialettica simbolica, la classe media è uno degli elementi migliori per noi per percepire come la sfera pubblica e privata fossero fondamentali per la loro autoaffermazione, riconoscimento e distinzione. Anche lontano dal concetto moderno di "meritocrazia", si[10] percepisce come l'archetipo dei più "ben adattati" riecheggia nell'immaginario del borghese medio. II merito ha sostituito il diritto di nascita come una nuova forma di differenziazione sociale, così come la raffinatezza intellettuale, un altro elemento che ha cominciato a distinguere la classe media dalle classi popolari, ancora senza accesso all'istruzione pubblica gratuita e di qualità.

La generalizzazione della famiglia della classe media, in cui la donna si prendecura dei suoi figli e delle faccende domestiche - controllando il lavoro di numerosi 
dipendenti - e l'uomo ha partecipato alle attività del mondo degli affari, è uno degli aspetti sorprendenti in questo senso, perché dà allindividuo la sensazione di continuità, vedendo nei suoi figli la possibilità di continuare con gli affari di famiglia. Pertanto, l'incorporazione di valori aristocratici, più che una semplice moda, faceva parte del processo di consolidamento dell'egemonia politica e sociale e della visione del mondo della classe media sulla societ[11]à.

Una volta stabilito l'universo simbolico borghese della classe media brasiliana, è chiaro la percezione antagonistica di questo verso gli altri gruppi meno ricchi e il loro tentativo di dotarsi simbolicamente delle élite, questi piuttosto veramente titolari dei mezzi di produzione e del controllo reale del paese. Questa ossessione di assomigliare alle élite non sarà, se non in alcuni casi, riconosciuta nella sfera del contante, quindi, la sua equivalenza simbolica per status soc[12]ale o prestigio rimane, e questo può essere ottenuto o riconosciuto attraverso tre elementi: occupazione produttiva (occupazione), consumo e stile di vita, tutti pienamente adatti alla società capitalista moderna, o, più di questo, uno che completa l'altro.

Con la conquista dello status nella moderna società meritocratica brasiliana, nuove forme di prestigio diventano parte della nuova gerarchia sociale. La cosa più importante per la classe media, oltre alla "virtù" dell'imitazione, è allontanarsi a vicenda dalle pratiche quotidiane di "scum" sociale. Bere vino a scapito della cachaàa, ascoltare musica classica invece di musica popolare, frequentare teatri, accademie letterarie e ristoranti costosi per l'esibizionismo del galateo, oltre al viaggio internazionale - con il diritto ai consigli di pseudointellettuali, nutrendosi dell'ambigua sensazione di repulsione a ciò che è nazionale - sono piccoli esempi di questa visione del mondo riduzionista.

\section{CAPITALE CULTURALE, ISTITUZIONI SOCIALI E PREGIUDIZI}

A differenza della visione culturalista individuata allinizio del nostro testo come un modo per spiegare le disuguaglianze sociali e le relazioni di classe, i concetti di soggettivazione e giustificare le differenze solo dal singolo punto di vista materiale, dobbiamo partire dalla comprensione sociologica delle istituzioni primarie e secondarie 
della socializzazione per comprendere il fenomeno dell'odio di classe così presente nella vita quotidiana.

In generale, la socializzazione inizia in famiglia, un'istituzione in cui l'individuo impara la lingua e, attraverso di essa, per comunicare, per esprimere i bisogni, così come i sentimenti di disagio o benessere. La ricerca psicologica come il piaget (2005) conferma l'importanza dei genitori e degli altri membri della famiglia nella formazione della personalità dei bambini. Questi studi indicano che all'inizio del processo di socializzazione i membri della famiglia tendono ad essere più decisivi degli individui con cui il bambino entra in contatto solo occasionalmente. Troviamo qui un problema: nell'organizzazione della società schiave brasiliana si è fatto tutto il possibile per evitare la costruzione di legami di solidarietà tra le popolazioni schiavizzate, e la prima istituzione ha combattuto è stata la famiglia, compromettendo questa fase della socializzazione. È interessante notare che non c'era poco spazio per la costituzione dei principali legami sociali in carcere, senza tenere conto dei bisogni psichici ed emotivi dei prigionieri.

La difficoltà dell'organizzazione familiare non è un semplice fenomeno del passato schiavo, ma rimane, nella proporzione, tra le popolazioni più umili, nei figli, nipoti e pronipoti delle precedenti generazioni di umiliante. Tutto ciò avrà profonde conseguenze nei futuri rapporti secondari, soprattutto nell'apprendimento scolastico, causando meno possibilità di prestigio nel mercato del lavoro. Le famiglie più deboli sono generalmente tassate come "disadattate" dalle classi superiori, in particolare dal discorso generalista della classe media, che, dalla culla, è stata in grado di costruire legami di solidarietà che saranno determinanti per il successo futuro.

L'attuale caso di sfruttamento della plebaglia brasiliana per risparmiare tempo dalle faccende domestiche sporche e pesanti - che consente di utilizzare il tempo "rubato" ad un prezzo vile in attività più produttive e ben retribuite - mostra una chiara funzionalità di miseria come la luce solare. Questa lotta silenziosa in classe esenta un'intera classe di assistenza all'infanzia e di vita familiare, trasformando il risparmio di tempo e l'apprendimento qualificante. La classe rubata, in questo caso, 
è condannata eternamente a svolgere gli stessi ruoli secolarmente servili. (Souza, 2019: 85).

Risparmia tempo sulle attività manuali e investi tempo nelle attività intellettuali. Di conseguenza, una maggiore formazione professionale e, di conseguenza, un maggiore prestigio sociale; si stabilisce un cerchio che non ha nulla a che fare con il merito, o la capacità personale, ma piuttosto con l'appropriazione di un maggiore capitale culturale.

Secondo Bourdieu (2004), i bambini provenienti da famiglie benestanti tendono ad avere prestazioni scolastiche più elevate rispetto ai giovani appartenenti a famiglie appartenenti alle classi popolari. Nella maggior parte dei casi, ciò è dovuto al fatto che gli studenti delle classi dominanti hanno un capitale culturale che gli altri non sono stati in grado di accumulare, non solo a scuola, ma anche nella formazione culturale dei genitori, che avrebbero insegnato ai loro figli le richieste di mantenere ed espandere la loro situazione sociale privilegiata. I bambini in situazioni di fragilità sociale non hanno questa rete di protezione, tendendo ad affrontare molte più difficoltà perché non hanno le conoscenze e le pratiche valorizzate dalla cultura dominante. Non parteciperebbero ai valori coltivati da queste élite e, per questo motivo, tenderebbero a considerare l'apprendimento della scuola una forma di violenza contro i loro valori e pratiche sociali, spiegando gli alti tassi di abbandono della scuola brasilian[13]a.

A scuola, possiamo avere la migliore percezione pratica della violenza simbolica. In esso, per esempio, è comune per gli studenti delle classi popolari imbattersi in una lingua, una pratica culturale e valori che non conoscono perché non hanno un certo patrimonio culturale, o, come sottolinea Bourdieu, un capitale culturale che permette loro di comprenderli o assimilarli, compromettendo le loro prestazioni scolastiche, diventando un impedimento imposto dall'istituzione stessa e delegittimando le prestazioni di questo studente. Nonostante questo, lo studente non si rende conto che il suo basso rendimento scolastico è determinato da fattori sociali e quindi spesso finisce per introdurre il fallimento, compromettendo la sua autostima in un momento cruciale della sua formazione non solo accademica ma umana. 
Avendo come punto di riferimento l'accesso e la continuità nel sistema educativo formale come una delle principali possibilità proclamate da una società che si considera "meritocratica", troviamo un sottile esempio di come i limiti imposti dal passato schiavo siano perpetuati, ma quasi invisibili. Se in passato potevamo percepire tutto esplicitamente disuguale, a causa del fenotipo e dell'esplicita esclusione degli schiavi alle istituzioni, oggi tutto sembra corretto a causa della creazione di un sistema di istruzione pubblica, spostando tutte le responsabilità di successo o di insuccesso verso la portata individuale. Copre il sole con il setaccio, pe[14]rché la maggior parte delle popolazioni bisognose sono escluse dal capitale culturale, deliberatamente appropriato dalla classe media e dalle élite, oltre ad avere accesso a un sistema di servizio pubblico inefficiente.

Nel mondo capitalista, la mancanza di successo materiale lo condanna allo status di fallimento. Immaginate allora di essere privi di capitale materiale e anche di capitale culturale? Poiché le forme di ascensione di classe nel sistema predatorio del capitale non sono facili, il ciclo riproduttivo della povertà e dell'esclusione tende a perpetuare dalla nascita a coloro che provengono dalle classi più povere. Questo si tradurrà in un soggetto, oltre a meno possibilità di costituire una formazione che gli permetterà di competere con i figli delle classi ricche, molto più propensi a subire tutti i tipi di violenza fisica e simbolica.

Poiché la riproduzione delle disuguaglianze di classe dalla culla viene repressa sia consapevolmente che inconsciamente, è lo stereotipo del nero, facilmente riconoscibile, che identifica facilmente il nemico da macellare e sfruttare. II "pericolo nero" usato come ricettario per massacrare indifesi e quilombola per secoli è continuato con altri mezzi nel massacro aperto, e oggi applaudito senza pejo, di poveri e neri nelle baraccopoli e nelle prigioni. E non solo. Poiché non c'era continuità temporale tra la schiavitù - che distrugge l'anima dall'interno, umilia e sminuisce il soggetto, rendendolo complice del proprio dominio - e la produzione di una plebaglia di disadattati al mondo moderno, i nostri 
esclusi ereditati, senza soluzione di continuità, ogni odio e vile disprezzo per i più deboli e con meno capacità di difendersi. (Souza, 2019: 88).

Ma perché tale disuguaglianza non sembra essere percepita dalla classe media brasiliana? Come abbiamo detto all'inizio di questo articolo, la teoria delle classi sociali prese dai criteri di reddito è insufficiente per comprendere una composizione sociale complessa come quella brasiliana, indebolita dalle conseguenze del suo passato schiavo. A nostro avviso è necessario superare il mero pregiudizio economico per entrare dalla storia delle mentalità[15]. Un cittadino della classe media tende a pensarlo come la sua classe, quindi non avrebbe alcuna base strumentale per la percezione dell'altro, rendendo l'esercizio dell'alterità infelice o spesso irraggiungibile. Questa assenza di dialettica produce persone prevenute, prive di percezione dell'altro, in un paese inserito nel XXI secolo all'interno di un ordine di mercato competitivo, che va oltre la sfera economica, raggiungendo la sociale, il pensiero e la percezione del mondo. II colonialismo mentale finisce per dominare le menti pur mantenendo lo status quo.

\section{CONSIDERAZIONI FINALI}

Tracciare una ricostituzione storica della formazione della classe media brasiliana e del suo archetipo di pregiudizio non è un compito facile a causa dell'ambiguità del concetto di classe sociale stessa. II fattore che crediamo sia essenziale per questa comprensione è la schiavitù passata, che interferisce drammaticamente nella comprensione del presente e del tessuto sociale brasiliano. È solo nel seriale a lungo termine che possiamo costruire la narrazione qui presentata e la nostra argomentazione per la comprensione delle classi dal contesto socioculturale, rompendo con l'economicismo tradizionale delle teorie di classe, a volte interpretate da pensatori liberali, a volte da pensatori marxisti.

Identificando l'importanza del passato schiavo per la configurazione delle mentalità di classe perpetuate dalle istituzioni sociali, possiamo percepire le disuguaglianze e gli stereotipi socialmente costituiti che sembrano riprodursi all'infinito. 
La famiglia, la scuola, le organizzazioni, tutto questo promuove nel corso della nostra storia la riproduzione dei privilegi dei gruppi elitari e della classe media, che si mimetizza con l'inchiostro di analisi pseudoscientifiche del meritocrazia, in uno dei paesi più disuguali del pianeta. L'errore che è possibile l'aumento quasi innato per alcuni a scapito di altri è stato incorporato nell'immaginario della classe media in modo così coerente che è riuscito a penetrare anche gli strati popolari, nel fenomeno che trattiamo nel testo circa l'introiezione del pregiudizio.

Essere nato nella classe media e medio-alta è quasi sinonimo della riproduzione del successo delle generazioni passate e dell'incorporazione di privilegi che faranno una differenza totale nel futuro. Sembrano fatti banali, ma acquisire fin dalla tenera età la disciplina dello studio, frequentare certi ambienti, vivere con persone con il capitale culturale necessario per eccellere nelle relazioni sociali, studiare una lingua straniera nel turno scolastico ed essere ben nutriti sono piccoli esempi di privilegi che, aggiunti a centinaia di altri, formeranno il futuro cittadino di successo, come se il suo merito personale fosse stato il grande differenziale di tale realizzazione.

Naturalizzare la violenza quotidiana contro le donne povere, nere, tra le altre minoranze, è sintomatico della violenza che ha plasmato la storia brasiliana. Gli echi della schiavitù si sentono in ogni morte nella favela, in ogni aggressione commessa dalla polizia, in ogni battuta a pranzo domenicale raccontata ai tavoli di famiglie che hanno già naturalizzato il razzismo come qualcosa di moralmente accettabile, come se non degradassero la dignità umana, trasformando così la realtà della classe media in una gabbia di paure, angoscia e odio sistemico.

\section{RIFERIMENTI}

BONFIM, Paulo Ricardo. Educar, higienizar e regenerar: uma história da eugenia no Brasil. São Paulo: Paco, 2019.

BOURDIEU, Pierre. A economia das trocas simbólicas. São Paulo: Perspectiva, 2004.

BRAUDEL, Fernand. Escritos sobre a história. São Paulo: Perspectiva, 1978. 
CARVALHO, José Murilo de. Cidadania no Brasil: o longo caminho. São Paulo: Civilização Brasileira, 2003.

DOWBOR, Ladislau. A era do capital improdutivo. São Paulo: Editora Novas Palavras, 2017.

FAUSTO, Bóris. História do Brasil. 6. ed. São Paulo: Edusp/FDE, 1997. . História concisa do Brasil. São Paulo :Edusp, 2002.

FERNANDES, Florestan. Revolução burguesa no Brasil: ensaio de interpretação sociológica. Rio de Janeiro: Zahar, 1974.

FROMM, Erich. Anatomia da destrutividade humana. Rio de Janeiro: Zahar, 1975.

KENNETH, Maxwell. A devassa da devassa. São Paulo: Paz e Terra, 1995.

KLEIN, Naomi. Sem logo: a tirania das marcas em um planeta vendido. Rio de Janeiro: Record, 2002.

PARSONS, Talcott. Social structure \& person. USA: Free Press, 2007.

PIAGET, Jean. A representação do mundo na criança. Curitiba: Ideias e Letras, 2005.

ROCHA, Sônia. Pobreza no Brasil. Rio de Janeiro: FGV, 2006.

SARTRE, Jean Paul. A prostituta respeitosa. Campinas: Papirus, 1992.

SOUZA, Jessé. A classe média no espelho. Rio de Janeiro: Estação Brasil, 2018. A elite do atraso. Rio de Janeiro: Estação Brasil, 2019.

SUCUPIRA, Eduardo. Introdução ao pensamento dialético. São Paulo: Alfa-Omega, 1984.

VOVELLE, Michel. Ideologias e mentalidades. São Paulo: Brasiliense, 1987. 
WEBER, Max. A ética protestante e o espírito do capitalismo. São Paulo: Companhia das Letras, 2007.

YOUNG, Michael. The rise of meritocracy. United Kingdom: Transaction Pub, 1994

\section{APPENDIX A}

\begin{tabular}{|c|c|c|c|c|c|c|c|c|c|c|}
\hline \multirow{3}{*}{ Grandes Regiões e car acteristicas selecionadas } & \multicolumn{10}{|c|}{$\begin{array}{l}\text { Taxa de frequência liquida a estabelecimento de ensino da população residente de } 6 \text { a } 24 \text { anos de idade, por grupos de idade e nivel de } \\
\text { ensino (\%) }\end{array}$} \\
\hline & \multicolumn{2}{|c|}{$\begin{array}{l}6 \text { a } 14 \text { anos, no ensino } \\
\text { fundamental }\end{array}$} & \multicolumn{2}{|c|}{$\begin{array}{l}6 \text { a } 10 \text { anos, nos anos } \\
\text { iniciais do ensino } \\
\text { fundamental }\end{array}$} & \multicolumn{2}{|c|}{$\begin{array}{l}11 \text { a } 14 \text { anos, nos anos } \\
\text { finais do ensino } \\
\text { fundamental }\end{array}$} & \multicolumn{2}{|c|}{$\begin{array}{l}15 \text { a } 17 \text { anos, no ensino } \\
\text { médio }\end{array}$} & \multicolumn{2}{|c|}{$\begin{array}{l}18 \text { a } 24 \text { anos, no ensino } \\
\text { superior }\end{array}$} \\
\hline & Taxa & $\mathrm{CV}(\%)$ & Taxa & $\mathrm{CV}(\%)$ & Taxa & $\mathrm{CV}(\%)$ & Taxa & $\mathrm{CV}(\%)$ & Taxa & $\mathrm{CV}(\%)$ \\
\hline Brasil & 97,0 & 0,1 & 95,5 & 0,2 & 85,9 & 0,3 & 68,5 & 0,6 & 23,2 & 1,3 \\
\hline Norte & 96,6 & 0,2 & 95,0 & 0,4 & 79,4 & 1,0 & 59,7 & 1,8 & 17,7 & 3,3 \\
\hline Nordeste & 96,8 & 0,1 & 95,3 & 0,2 & 82,0 & 0,6 & 60,7 & 1,1 & 16,8 & 2,9 \\
\hline Sudeste & 97,3 & 0,2 & 95,7 & 0,3 & 89,2 & 0,5 & 76,5 & 1,0 & 25,8 & 2,4 \\
\hline Sul & 97,4 & 0,2 & 96,1 & 0,3 & 89,6 & 0,6 & 69,6 & 1,4 & 29,1 & 2,4 \\
\hline Centro-Oeste & 96,8 & 0,3 & 95,0 & 0,4 & 88,4 & 0,9 & 70,4 & 1,7 & 30,1 & 2,9 \\
\hline \multicolumn{11}{|l|}{ Situaçäo do dom icilio } \\
\hline Urbana & 97,1 & 0,1 & 95,5 & 0,2 & 87,2 & 0,3 & 70,6 & 0,7 & 25,6 & 1,4 \\
\hline Rural & 96,9 & 0,2 & 95,6 & 0,2 & 79,6 & 0,7 & 57,4 & 1,3 & 8,3 & 3,5 \\
\hline \multicolumn{11}{|l|}{ Sexo } \\
\hline Homem & 96,9 & 0,1 & 95,3 & 0,2 & 83,5 & 0,5 & 63,6 & 0,9 & 19,7 & 1,8 \\
\hline Mulher & 97,2 & 0,1 & 95,7 & 0,2 & 88,3 & 0,4 & 73,7 & 0,7 & 26,8 & 1,5 \\
\hline \multicolumn{11}{|l|}{ Cor ou raça (1) } \\
\hline Branca & 97,3 & 0,1 & 95,8 & 0,2 & 89,5 & 0,4 & 76,6 & 0,8 & 32,9 & 1,6 \\
\hline Preta ou parda & 96,9 & 0,1 & 95,3 & 0,2 & 83,7 & 0,4 & 63,5 & 0,9 & 16,7 & 1,8 \\
\hline \multicolumn{11}{|l|}{$\begin{array}{l}\text { Classes de percentual de pessoas em ordem } \\
\text { crescente de rendimento domiciliar per capita }\end{array}$} \\
\hline Até $20 \%$ & 96,6 & 0,2 & 94,9 & 0,2 & 78,9 & 0,6 & 54,7 & 1,3 & 6,6 & 4,5 \\
\hline Mais de $20 \%$ até $40 \%$ & 96,9 & 0,2 & 95,4 & 0,3 & 85,3 & 0,6 & 65,3 & 1,2 & 12,0 & 3,3 \\
\hline Mais de $40 \%$ até $60 \%$ & 97,5 & 0,2 & 96,1 & 0,3 & 90,1 & 0,6 & 73,9 & 1,0 & 20,1 & 2,6 \\
\hline Mais de $60 \%$ até $80 \%$ & 97,5 & 0,3 & 95,9 & 0,5 & 93,0 & 0,6 & 81,1 & 1,2 & 32,3 & 2,1 \\
\hline Mais de $80 \%$ & 97,8 & 0,3 & 96,1 & 0,5 & 95,0 & 0,5 & 90,7 & 0,8 & 58,3 & 1,5 \\
\hline
\end{tabular}

Fonte: IBGE Pesquisa Nacional por Amostra de Domicilios Continua, 2017, segundo trimestre.

Nota: (1) Não são apresentados resultados para amarelos, indigenas e pessoas sem declaração de cor ou raça 


\section{APPENDIX B}

\begin{tabular}{|c|c|c|c|c|c|c|c|c|c|c|c|c|}
\hline \multirow{4}{*}{$\begin{array}{l}\text { Grandes Regiōes e } \\
\text { Unidades da Fede raçäo }\end{array}$} & \multicolumn{12}{|c|}{ Distribuição percentual da população, por classes de percentual de pessoas em ordem crescente de rendimento real efetivo domiciliar per capita e cor ou raça (\%) } \\
\hline & \multicolumn{4}{|c|}{ Total } & \multicolumn{4}{|c|}{ Entre os $10 \%$ com menores rendimentos } & \multicolumn{4}{|c|}{ Entre os $10 \%$ com maiores rendimentos } \\
\hline & \multicolumn{2}{|c|}{ Branca } & \multicolumn{2}{|c|}{ Preta ou parda } & \multicolumn{2}{|c|}{ Branca } & \multicolumn{2}{|c|}{ Preta ou parda } & \multicolumn{2}{|c|}{ Branca } & \multicolumn{2}{|c|}{ Preta ou parda } \\
\hline & Percentual & CV (\%) & Percentual & CV (\%) & Percentual & CV (\%) & Percentual & CV (\%) & Percentual & CV (\%) & Percentual & CV (\%) \\
\hline Brasil & 43,6 & 0,5 & 55,4 & 0,4 & 23,9 & 1,8 & 75,2 & 0,6 & 71,7 & 0,8 & 26,3 & 2,3 \\
\hline Norte & 20,1 & 1,9 & 78,4 & 0,5 & 12,5 & 6,8 & 84,8 & 1,3 & 34,2 & 3,8 & 64,4 & 1,9 \\
\hline Rondônia & 31,2 & 3,0 & 68,2 & 1,4 & 23,6 & 9,3 & 75,2 & 2,9 & 40,9 & 7,9 & 58,4 & 5,5 \\
\hline Acre & 19,1 & 4,3 & 79,9 & 1,1 & 14,1 & 12,5 & 85,3 & 2,1 & 31,8 & 8,2 & 67,7 & 3,8 \\
\hline Amazonas & 18,1 & 4,7 & 79,5 & 1,2 & 11,0 & 13,1 & 86,2 & 2,0 & 38,2 & 9,8 & 60,5 & 5,8 \\
\hline Roraima & 21,5 & 5,6 & 72,4 & 2,0 & 14,6 & 17,7 & 71,3 & 7,3 & 40,9 & 8,4 & 55,5 & 6,5 \\
\hline Pará & 18,2 & 3,3 & 80,6 & 0,8 & 11,8 & 12,6 & 85,8 & 2,2 & 30,0 & 5,5 & 68,9 & 2,4 \\
\hline Amapá & 19,3 & 6,5 & 80,3 & 1,6 & 10,6 & 26,6 & 87,7 & 3,6 & 25,9 & 13,4 & 73,4 & 4,8 \\
\hline Tocantins & 23,7 & 4,7 & 75,3 & 1,5 & 14,8 & 19,0 & 84,4 & 3,4 & 42,5 & 6,4 & 56,2 & 4,7 \\
\hline Nordeste & 24,8 & 1,2 & 74,6 & 0,4 & 18,7 & 3,3 & 80,8 & 0,8 & 42,8 & 2,7 & 56,4 & 2,0 \\
\hline Maranhão & 18,5 & 2,6 & 80,8 & 0,6 & 15,7 & 6,9 & 83,9 & 1,3 & 31,5 & 5,5 & 67,4 & 2,6 \\
\hline Pauí & 20,5 & 4,8 & 79,4 & 1,2 & 18,6 & 11,0 & 81,2 & 2,5 & 40,9 & 7,6 & 58,6 & 5,5 \\
\hline Ceará & 27,5 & 2,2 & 71,9 & 0,8 & 20,4 & 6,2 & 79,1 & 1,6 & 46,9 & 4,3 & 52,6 & 3,8 \\
\hline Rio Grande do Norte & 36,7 & 2,6 & 63,1 & 1,5 & 30,6 & 7,1 & 69,1 & 3,1 & 50,9 & 5,5 & 48,8 & 5,8 \\
\hline Paraba & 33,8 & 3,0 & 66,1 & 1,5 & 30,1 & 7,2 & 69,8 & 3,1 & 54,1 & 5,4 & 45,7 & 6,3 \\
\hline Pernambuco & 30,7 & 3,0 & 68,5 & 1,4 & 23,2 & 8,3 & 76,5 & 2,5 & 51,5 & 5,8 & 47,4 & 6,2 \\
\hline Alagoas & 23,4 & 2,8 & 76,1 & 0,9 & 21,8 & 6,3 & 77,9 & 1,8 & 36,1 & 6,6 & 63,5 & 3,8 \\
\hline Sergipe & 20,8 & 5,4 & 78,3 & 1,4 & 13,6 & 13,9 & 85,7 & 2,2 & 36,6 & 12,4 & 61,7 & 7,3 \\
\hline Bahia & 19,2 & 3,9 & 80,2 & 0,9 & 14,7 & 9,4 & 84,6 & 1,6 & 35,3 & 9,1 & 63,6 & 4,9 \\
\hline Sudeste & 51,2 & 0,9 & 47,6 & 1,0 & 35,2 & 2,8 & 64,2 & 1,5 & 78,1 & 1,1 & 19,0 & 4,6 \\
\hline Minas Gerais & 40,4 & 1,7 & 59,3 & 1,2 & 24,5 & 5,5 & 75,2 & 1,8 & 64,8 & 2,6 & 34,8 & 4,8 \\
\hline Espirito Santo & 39,2 & 2,6 & 60,2 & 1,7 & 22,5 & 10,6 & 77,2 & 3,1 & 64,5 & 3,0 & 35,2 & 5,6 \\
\hline Rio de Janeiro & 43,8 & 1,7 & 55,7 & 1,3 & 28,4 & 5,2 & 71,2 & 2,1 & 74,5 & 1,9 & 24,8 & 5,8 \\
\hline São Paulo & 60,1 & 1,2 & 38,1 & 2,0 & 46,8 & 3,7 & 52,4 & 3,3 & 83,1 & 1,4 & 12,3 & 9,0 \\
\hline Sul & 75,6 & 0,5 & 23,7 & 1,7 & 59,3 & 2,1 & 39,9 & 3,2 & 91,3 & 0,6 & 7,9 & 6,9 \\
\hline Paraná & 67,2 & 1,1 & 31,7 & 2,3 & 52,2 & 3,8 & 46,7 & 4,3 & 86,7 & 1,3 & 11,4 & 9,4 \\
\hline Santa Catarina & 82,8 & 0,7 & 16,9 & 3,4 & 68,5 & 2,9 & 31,2 & 6,4 & 92,8 & 0,9 & 6,8 & 11,7 \\
\hline Rio Grande do Sul & 79,6 & 0,9 & 20,0 & 3,5 & 64,2 & 3,3 & 35,2 & 6,1 & 94,4 & 0,9 & 5,5 & 15,4 \\
\hline Centro-Oeste & 36,3 & 1,4 & 62,6 & 0,8 & 25,0 & 4,5 & 74,0 & 1,5 & 56,2 & 3,4 & 41,0 & 4,0 \\
\hline Mato Grosso do Sul & 43,2 & 2,6 & 55,5 & 2,1 & 28,9 & 8,7 & 69,1 & 3,7 & 65,0 & 3,6 & 34,1 & 6,8 \\
\hline Mato Grosso & 31,8 & 3,2 & 67,0 & 1,5 & 23,7 & 12,3 & 74,9 & 3,9 & 54,4 & 4,8 & 43,9 & 6,0 \\
\hline Golás & 35,7 & 2,2 & 63,6 & 1,2 & 23,9 & 7,4 & 75,8 & 2,3 & 51,6 & 4,2 & 47,7 & 4,6 \\
\hline Distrito Federal & 36,7 & 4,1 & 61,6 & 2,4 & 22,8 & 11,0 & 76,5 & 3,3 & 59,3 & 10,5 & 29,1 & 10,0 \\
\hline
\end{tabular}

\section{APPENDIX C - RIFERIMENTI FOOTNOTE}

2. Para o entendimento da financeirização do capitalismo e suas repercussões no mundo do trabalho, vide DOWBOR, Ladislau. A era do capital improdutivo. São Paulo: Editora Novas Palavras, 2017.

3. SARTRE, Jean Paul. A prostituta respeitosa. Campinas: Papirus, 1992. In questo pezzo, l'autore ritrae come l'introiezione del pregiudizio, incorporata dalla vittima, sia naturalizzata all'interno della società degli Stati Uniti meridionali.

4. BRAUDEL, Fernand. Escritos sobre a história. São Paulo: Perspectiva, 1978.

5. FROMM, Erich. Anatomia da destrutividade humana. Rio de Janeiro: Zahar, 1975. 
6. Uma das obras mais consistentes e rica em fontes primárias sobre a organização das regiões mineradoras é de KENNETH, Maxwell. La dissolutezza della dissolutezza. São Paulo: Paz e Terra, 1995.

7. Sobre o período em questão, vide FAUSTO, Bóris. História concisa do Brasil. São Paulo: Edusp, 2002.

8. Para um melhor entendimento sobre a construção do processo da cidadania brasileira, vide CARVALHO, José Murilo de. Cidadania no Brasil: o longo caminho. São Paulo: Civilização Brasileira, 2003.

9. Abbiamo usato metaforicamente l'espressione "palla di neve nuda", perché i neri, meticci, indigeni, marroni, oltre naturalmente alla popolazione bianca emarginata, addensavano il contingente di "scorie" segregate dalla società gerarchica brasiliana. Durante il XIX secolo, il razzismo scientifico e i principi dell'eugenetica presenti in America, in particolare negli eugenetici americani, i suoi principali portavoce. Anche se il pregiudizio in Brasile è erroneamente limitato a questioni fenotipiche, il ruolo svolto dagli eugenetici nello stimolare le politiche di sbiancamento, volutamente assunto dal governo brasiliano per purificare la genetica della popolazione, è considerevole. Ciò ha contribuito direttamente allo stimolo al razzismo e all'animalizzazione degli individui considerati "inferiori". BONFIM, Paulo Ricardo. Educar, higienizar e regenerar: uma história da eugenia no Brasil. São Paulo: Paco, 2019.

10. In Brasile, il concetto di "meritocrazia" permea il buon senso e la demagogia. A nostro avviso, si intende solo la "meritocrazia" se si entra nella sua origine nella letteratura in cui il concetto nasce in modo satirico e ironico. Ver YOUNG, Michael. The rise of meritocracy. United Kingdom: Transaction Pub, 1994.

11. "Con l'avvento della cultura di massa nel ventesimo secolo, il capitalismo crea un nuovo simbolismo di falsa inclusione, introducendo nella società contemporanea quella che chiamiamo la "soppressione della soggettività", sollevando lo sviluppo del cosiddetto "comportamento mimetico", cioè, l'individuo avrebbe smesso di sviluppare una propria personalità, originale, che lo metterebbe in conflitto e tensione con la realtà 
sociale consolidata". KLEIN, Naomi (2009). Sem logo: a tirania das marcas em um planeta vendido. Rio de Janeiro: Record, 2002.

12. Tomamos como referência o conceito de status em WEBER, Max. Etica protestante e spirito del capitalismo. São Paulo: Companhia das Letras, 2007.

13. Secondo le Appendici A e B, possiamo notare chiaramente i mali storici del processo di esclusione delle popolazioni nere e marroni nel loro percorso educativo, allontanando questi gruppi dai meccanismi di accesso richiesti dalla società contemporanea per una vita dignitosa, che richiede un migliore inserimento professionale, compromettendo i loro redditi e la loro capacità di entrare nel mondo capitalista.

14. Espressione popolare brasiliana usato per descrivere misure inefficaci per alcuni problemi.

15. VOVELLE, Michel. Ideologias e mentalidades. São Paulo: Brasiliense, 1987.

Inviato: Dicembre 2019.

Approvato: febbraio 2020. 